\title{
Enterprise Credit Evaluation Model Based on Genetic Algorithm Optimization BP Neural Network
}

\author{
Yu-jing Zhang ${ }^{12}$, Qian $\mathrm{Li}^{1}$,Zhi-wang Jiang ${ }^{12}$ and Hong-xia Zhang ${ }^{1}$ \\ ${ }^{1}$ Department of Information Management and Engineering, Hebei Finance University, Baoding, \\ 071000, China \\ ${ }^{2}$ Science and Technology Financial Key Laboratory of Hebei Province, Baoding, 071000, China
}

Key words: Power supply enterprise; Credit evaluation; Genetic algorithm; BP neural network

\begin{abstract}
Credit is very important for the enterprise, analysis of a comprehensive evaluation of enterprise credit, can enhance the risk control ability of enterprise credit, improve enterprise credit rating.The paper establishes BP neural network credit evaluation model based on genetic algorithm

(GA) optimization ,and to the power supply enterprise credit evaluation as an example, to verify the practicability of the model in the evaluation of enterprise credit. Examples of verification results indicate that the genetic algorithm (GA) to optimize the BP neural network is better than traditional BP neural network, it's evaluation has higher accuracy, stronger generalization ability, more suitable for the enterprise credit evaluation.
\end{abstract}

\section{Introduction}

The evaluation of enterprise credit through fast, timely, authoritative information disclosure, to reduce information asymmetry between operators and investors, to avoid the moral risk of enterprise managers[1].

With the deepening of electrical power system reforms, the effect of power supply enterprises credit is growing, which impacts not only the image of enterprises, but also the enterprises' survival and development. Therefore, in order to gradually increase the capability of the power supply enterprise credit risk control, it is required to carry on a comprehensive evaluation of the electricity supply enterprises credit. How to use scientific evaluation methods, and effectively improve the credit rating of power supply enterprise has become a necessary part of business management.So this paper takes credit evaluation of power supply enterprise as an example, to verify the genetic algorithm for optimization of neural network is suitable for suitable for enterprise credit evaluation[2].

\section{Credit risk evaluation system of electricity supply enterprises}

The establishment of evaluation index system of credit is a prerequisite to evaluate the credit of enterprise, through a comprehensive evaluation of each factor in the index system, To measure the different enterprise's credit rating situation [3].The credit evaluation index system of enterprise such as shown in Table 1.

\section{Neural network}

BP (Back Propagation) network is a group of scientists led by Rumelhart and McCelland put forward in 1986 , is a kind of error back-propagation algorithm for training multilayer feedforward network, its learning rule is to use the method of steepest descent, to constantly adjust the network weight value and threshold value by back propagation, make the network and the minimum sum of square error. BP neural network is mainly composed of an input layer, one or more hidden layers and one output layer, the mutual connection between the layers of neurons, but between each layer between the neurons are not connected relationship. 
Table 1 Credit evaluation index system of the enterprise

\begin{tabular}{|c|c|c|}
\hline \multirow{12}{*}{$\begin{array}{l}\text { Credit } \\
\text { evaluation } \\
\text { index } \\
\text { system of } \\
\text { the } \\
\text { enterprise }\end{array}$} & \multirow{4}{*}{$\begin{array}{c}\text { Management Ability } \\
\text { Evaluation Indicator } \\
\qquad P_{1}-P_{4}\end{array}$} & Human Resources $P_{1}$ \\
\hline & & Business Structure $P_{2}$ \\
\hline & & Technical Capacity $P_{3}$ \\
\hline & & Scale and Efficiency $P_{4}$ \\
\hline & \multirow{4}{*}{$\begin{array}{l}\text { Management Ability } \\
\text { Evaluation Indicator } \\
P_{5}-P_{8}\end{array}$} & Management Quality $P_{5}$ \\
\hline & & Enterprise Culture $P_{6}$ \\
\hline & & Operational Performance $P_{7}$ \\
\hline & & Social Responsibility $P_{8}$ \\
\hline & \multirow{4}{*}{$\begin{array}{l}\text { Financial Strength } \\
\text { Evaluation Indicator } \\
P_{9}-P_{12}\end{array}$} & Total Assets $P_{9}$ \\
\hline & & Profitability $P_{10}$ \\
\hline & & Operating Capacity $P_{11}$ \\
\hline & & Solvency $P_{12}$ \\
\hline
\end{tabular}

The learning process of the BP neural network model composed of by two parts,forward and backward .In the forward process of communication, information from the input layer through the middle hidden layer to the output layer weighted propagation, in the output layer to obtain the input of the network response, output value by comparing the function calculation and target output value, if there are errors, error back propagation along the route before the return, i.e., from the output layer after each intermediate the hidden layer to adjust the connection weights, finally back to the output layer, to reduce the error, with the neural network error reverse spread to revise the weights continuously, the entire network accuracy of input information in response to natural also improved.

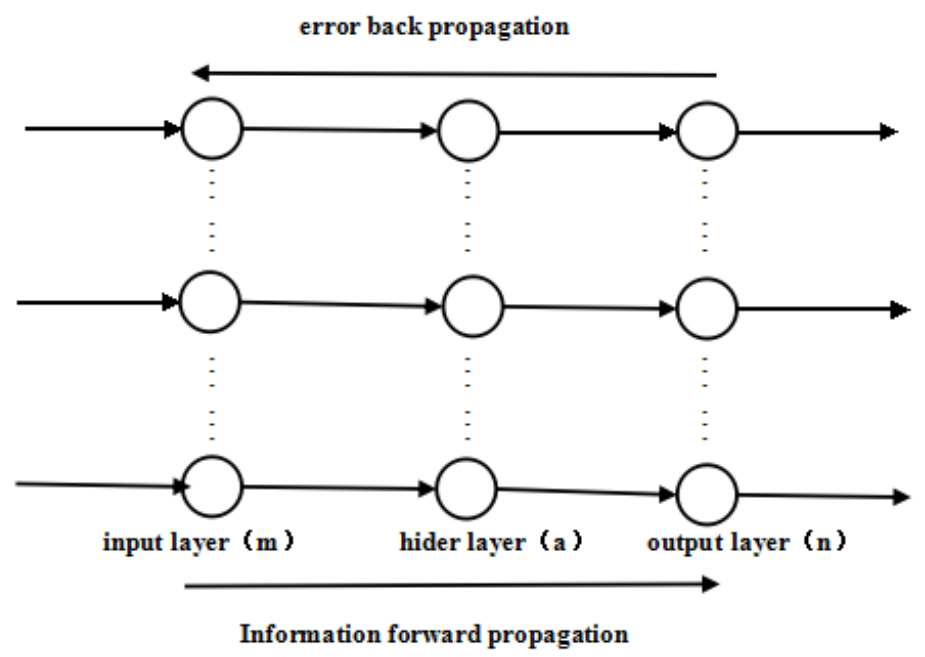

Figure 1.A classical 3-layer BP network model

\section{Genetic algorithm (GA) optimize BP neural network}

The operation object of GA is a population, and each population is composed of several chromosomes. Each chromosome corresponds to a solution. Start from an initial population, use replication, crossover and mutation to produce the next generation of population. Finally the optimal solution is got to meet certain convergence conditions [11]. BP network mode of transmission is divided into two kinds which are forward propagation and back propagation. In the forward propagation, the input information transmits from the input layer to the output layer after the hidden layer processing. The state of the neuron in each layer only affects the state of the next layer neurons. In the output layer, after making a contrast between the actual output and expected output, the operation turns to the back-propagation and the error signal returns along the original 
neurons if the actual output does not meet the requirements. In the return process, the weights linking each layer are modified one by one. This process is continuous. Finally the error is within the scope allowed. Its mathematical description as follows [12]:

$$
\left\{\begin{array}{l}
\min E_{1}(\boldsymbol{w}, \boldsymbol{v}, \theta, r)=\frac{1}{2} \sum_{k=1}^{M} \sum_{t=1}^{n}\left[y_{k}(t)-\hat{y}_{k}(t)\right]^{2} \\
s, t: \boldsymbol{w} \in R^{m \times p}, \boldsymbol{v} \in R^{n \times p}, \theta \in R^{p}, r \in R^{n}
\end{array}\right.
$$

$E_{1}$ is the total network error, $y_{k}(t)$ is the desired output, and $\hat{y_{k}}(t)$ is the actual network output.

$$
\begin{aligned}
& \hat{y}_{k}(t)=f\left\{\sum_{j=1}^{p} v_{j t} \cdot f\left[\sum_{i=1}^{m} w_{i j} \cdot x_{i}(t)+\theta_{j}\right]\right\}+r_{t} \\
& E_{2}=\frac{1}{N-N_{1}} \sum_{k=N_{1}}^{N} \sum_{t=1}^{n}\left[y_{k}(t)-\hat{y}_{k}(t)\right]^{2}
\end{aligned}
$$

$E_{2}$ is the mean square error of the test sample, which means the estimate of the output data reliability. The smaller $E_{2}$ is, the more reliable network output is.

In the BP neural network, the weights initialization strongly affect the final solution. The initial weights of different settings may cause great differences on the training time and convergence. In order to better solve the problems of determine the initial weights and threshold, can adopt the weights and threshold based on genetic algorithm, using the global search ability of genetic algorithm to determine the initial weights and threshold. This paper adopts three layer BP network to determine the initial solution space, setting training number and training error of the network. when the training sample error and testing samples are more satisfied, respectively recorded maximum value and minimum value of the connection weights as $w_{\max }$ and $w_{\min }$. Set the solution space of the connection weights as $\left[w_{\min }-\delta_{1}, w_{\max }+\delta_{2}\right] \quad\left(\delta_{1}, \delta_{2}\right.$ is adjusting parameters). The chromosome representation the weights, then the fitness function is the calculation error of the neural network, and the error is larger, the fitness is smaller. The GA fitness function can be use of

$$
\text { fitness }=\frac{1}{\frac{1}{N} \sum\left[y_{k}(t)-\hat{y}_{k}(t)\right]^{2}}
$$

$y_{k}(t)$ and $\hat{y}_{k}(t)$ are the expected value and the actual value of the network output. $\mathrm{N}$ is constant.Set the input population size, crossover probability $\left(P_{c}\right)$, mutation probability $\left(P_{m}\right)$, the network layers, each layer neural metadata, and use GA to optimize the weights of neural network repeatedly, until the average value is no longer meaningful increase so far, at this time the decoded parameter combination has sufficiently close to the optimum combination of parameters, and then $\mathrm{BP}$ algorithm reoptimization connection weights and threshold of the network in the small solution space, search out the optimal solution[4].

Because GA is based on the population, not to search base on a single point, can also obtain a plurality of extreme value from different points, so it is not easy to fall into local optimum, which can effectively solve the existing problem in BP neural network, and effectively improve the generalization performance of neural network.

\section{Empirical Analyses}

(1) Sample selection

This paper selects 40 power supply enterprises of raw data, and then select the relevant experts in the field according to the table 2 shows the credit grade evaluation criteria for scoring, obtain the initial sample data, then use the attribute reduction of rough set to the initial sample, finally obtained the sample data as shown in Table 3 . 
Table 2 Credit level standard of enterprise

\begin{tabular}{lllllll}
\hline $\begin{array}{l}\text { Credit } \\
\text { level } \\
\begin{array}{l}\text { standar } \\
\text { d }\end{array}\end{array}$ & AAA & AA & A & B & C & D \\
\hline Score & $100-90$ & $90-80$ & $80-70$ & $70-60$ & $60-50$ & $50-0$ \\
\hline
\end{tabular}

Table 3 Index after reduction

\begin{tabular}{|c|c|c|c|c|c|c|c|c|c|c|c|c|}
\hline & P1 & P2 & P3 & P4 & P5 & P6 & P7 & P8 & P9 & P10 & P11 & P12 \\
\hline sample 1 & 86.9 & 87.4 & 88 & 87.7 & 88 & 85.8 & 87 & 88.5 & 87 & 90 & 86.5 & 84.6 \\
\hline sample 2 & 87 & 87.8 & 88.5 & 87.5 & 89 & 86 & 86.7 & 89.2 & 86.8 & 90.5 & 86.8 & 85 \\
\hline sample 3 & 85.5 & 87.5 & 88.1 & 88.5 & 89.7 & 86.2 & 86.5 & 89.7 & 86.5 & 89.6 & 86 & 87 \\
\hline sample 4 & 85.9 & 87.6 & 87.6 & 89 & 88.4 & 86.1 & 86 & 89 & 87 & 89.1 & 86.1 & 86.2 \\
\hline & & & & & & & & & & & & \\
\hline sample 36 & 87.2 & 87 & 87.5 & 88.6 & 85.6 & 86.5 & 88.3 & 86 & 89.5 & 86.3 & 86.4 & 85 \\
\hline sample 37 & 88.1 & 88 & 87.8 & 88.7 & 86 & 85.8 & 88 & 87 & 89 & 86.4 & 84.8 & 84.4 \\
\hline sample 38 & 87.8 & 87.6 & 87.2 & 88.5 & 86.2 & 85.8 & 88.7 & 87.2 & 89.2 & 86.9 & 85.5 & 84.6 \\
\hline sample 39 & 87.8 & 88.2 & 88.5 & 88.6 & 85.7 & 85.6 & 88.4 & 87.2 & 89.6 & 87 & 85.4 & 84.8 \\
\hline sample 40 & 87.5 & 87.4 & 88.1 & 89 & 85.1 & 86 & 88.8 & 86.4 & 89.4 & 85.5 & 85.6 & 84.6 \\
\hline
\end{tabular}

(2) Model training and results analysis

The first 35 samples as training samples, the last 5 samples as test samples, Use of neural network model optimized by genetic algorithm training the sample data, the training results as shown in table 4.

Tab.4 Contrast of expect output and the actual output

\begin{tabular}{llllll}
\hline & 36 & 37 & 38 & 39 & 40 \\
\hline $\begin{array}{l}\text { Expected } \\
\text { Output }\end{array}$ & 91.11 & 81.44 & 71.18 & 68.00 & 51.30 \\
Actual & 91.1106 & 81.4401 & 71.1796 & 68.0001 & 51.2995 \\
Output & 7 & 2 & 7 & 7 & 4 \\
Error & 0.00067 & 0.00012 & -0.0003 & 0.00017 & -0.0004 \\
& & & 3 & & 6 \\
Credit & AAA & AA & A & B & C \\
Rating & & & & &
\end{tabular}

From table 4, we can see that the error model of the actual output and the expected output value is very small, less than 0.001 . And in accordance with the actual situation of enterprise credit rating. This indicates that BP neural network optimized by GA has a more accurate evaluation results in the aspects of enterprise credit evaluation.

\section{Conclusion}

Credit evaluation is of great significance for companies. This paper presents a genetic algorithm which optimize the initial values of weights and thresholds of BP neural network algorithm. It cleverly and reasonably combines the neural network error function and fitness function. GA-BP overcomes the defects of the traditional methods, and can display BP network's searching ability within a small scale precision. It overcomes the network oscillation because of the randomness, which is produced in the determination process of traditional neural network topology and initial weights. It avoids local optimization, accelerates the computational speed of the neural network and can quickly make accurate business credit evaluation with strong operability.

\section{Acknowledgements}

This research is supported by the natural science key project of Hebei Education Department(ZD20131083), the Youth Fund project of Hebei Education Department(QN2014075), the Youth Fund project of Hebei Education Department(Q2012052), and the key developing 
discipline of computer application technology in Hebei Finance University of Hebei Province.

\section{References}

[1] Wei Sun, Qing Ren. BP Neural Network Application in Wind Turbine Type Selection Based on Particle Swarm Optimization[J].Journal of Information and Computational Science (JICS),2014,11(7): 2415-2423

[2] Lei Huang,Shu-bi Zhang,Qiu-zhao Zhang .Application of particle swam optimization BP neural network to GPS elevation fitting[J].Journal of Geomatics,34（6）,2009,18-19.

[3] Jia-yang Wang ,Chun Guo,Zuo-yong Li .Preliminary evaluation model of mine safety based on neural networks optimized by particle swarm optimization[J].Journal of Computer Applications,30 (s1) ,2012,74-75.

[4] Dong-Xiao Gu, Chang-Yong Liang, Isabelle Bichindaritz,A case-based knowledge system for safety evaluation decision making of thermal power plants[J].Knowledge-Based Systems, 2012, 26 (2) : 185-195

[5] Li Xin,Yu, Yuan-Yuan; Liu, Yan.Research on safety evaluation about foundation excavation of subway stations based on fuzzy theory[J].Shenyang Jianzhu Daxue Xuebao ( Natural Science) ,2006, 22(3):375-378

[6] CHAI Yi,YIN Hong-peng,LI Da-jie. BP neural network adaptive optimization design based on the improved genetic algorithm[J]. Chongqing university journal, 2007,30(4):91-96.

[7] LI Yong,WANG Jian-jun,CAO Li-hua. Real time optimal load dispatch of power plant based on back propagation neural network[J]. Power System Protection and Control, 2011, 39(17):87-92.

[8] GAO Xian-jun,ZHANG Jie,HONG Yu. Neural network technology research Based on genetic algorithm[J]. Equipment manufacturing technology, 2010,2(2):8-10.

[9] ZHU Wen-long.Application research of BP neural network in Multi-objective optimization based on genetic algorithm[D]. Harbin: Harbin university of technology,2009. 